\title{
Specified Target Detection and Tracking on Complex Infrared Background
}

\author{
Sun Jingle, Tang Linbo, Zhao Baojun, Liu Qing \\ Information and Electronic College \\ Beijing Inst. Of Technology
}

\begin{abstract}
In this paper, we analyze the key problems in the tracking of the specified target on the complex infrared background, for example how to judge the polarity of target, how to select the seed of region growing, and how to determine the suitable size of the detection window. We propose a reasonable solution for each problem, such as to judge the target polarity by comparing the gray value, and to segment the target by using the adaptive region growing and then determine the size of detection window. In this $p$, aper, we propose the spokes variance (SV) method to describe the shape, and use the features of target to exclude interference, which include area, location, gray value and shape feature. Through the Kalman Filter to predict position of target, and solve the problem of target being covered in short time. The experimental results show that the algorithm is simple and effective, and achieved good tracking effect.
\end{abstract}

Keywords-complex infrared background, specified target, region growing, spokes variance (SV), Kalman Filter

\section{INTRODUCTION}

Target detection and tracking on the complex background is always the difficult problem of the image field, there are much interference caused by complex background, it will bring higher false alarms and missed detection probability with automatic target detection, it is an effective solution to specify a target artificially in practical engineering. But the specified target only give rough information of target position, the size of the target area is unknown, so some of the common methods are no longer applicable, such as template matching, thresholding and Otsu, et al. The key of the template matching is to select the appropriate template, the principle to get a template is to contain the background as little as possible on the condition of including complete target, because the exact target region can not been get in advance, it is difficult to guarantee the quality of the template, on the other hand template will drift when it is updated dynamically[1], the longer of tracking time, the more drift of the template. On the complex background, both thresholding and Otsu's[2, 3] segmentation results are not ideal. Particle filter and mean shift methods are too complex to meet the requirement of real-time tracking[4, 5]. In this paper, we use region growing to segment target[6-8], the reason is this algorithm don't need the target boundary information, but need the location of seeds that can be get from coordinate of specified target, region growing will stop to grow when no pixel satisfies with the growing rules, so region growing method is adapt to segment target on the complex background. We recognize target by combined with several target features, such as target size, location, intensity and shape. We propose the SV method to describe shape feature. To resolve the problem of tracking target which was covered in a short time through using Kalman Filter to predict target trajectory. Experiments show that the algorithm can achieve good tracking effect.

\section{REGION GROWING}

The thought of region growing is to collect pixels with similar properties together and constitute a region in which all pixels has the maximum consistency[8]. Firstly, the method need to specify a seed pixel as a starting point for growth, and then merge the others pixel that around it and has similar property to the region which the seed in it, until there no pixels to meet the growing rules. When growth stopped and a region is formed. And then give another seed pixel to repeat the above steps, until all the pixels in the image are grew.

The region growing rules, which is the condition to stop grow, will result in the different growing result. We make the growing rules as follow:

$$
\left|I_{s}-I\right|<\lambda\left|I_{\max }-I_{\min }\right|
$$

Where, $I_{s}$ is the gray value of seed pixel, $I$ is the gray value of pixel will be grew. $I_{\max }, I_{\min }$ denote the maximum and the minimum gray value respectively in the detecting window. $\lambda$ is a positive fraction less than 1 , if its value is too big, it will lead to the region over-growth, but too small it will easy to create growth and value is not sufficient.

\section{KEY PROBLEM SOLVED IN FIRST FRAME}

When target is specified, we know the rough target location only, but the target polarity, which denote the target is bright or dark, and area are unknown. Generally, it is often to open a window, the purpose is to avoid the target to be interfered and reduce computing time, so we should determine the size of window during the first frame. As the position of specified target can not guarantee to be the brightest point in the local area, if take it as the growing seed directly, the growing result will be unstable, so the seed point need to be adjusted. According to all of above, there are three important tasks to do during the first frame: to judge the target polarity, to adjust the seed point and to determine the size of the window.

\section{A. Judge target polarity}

Getnerally, the mechanism of infrared image is that the temperature is higher, the image infrared image if brighter. But sometimes the mechanism is in reverse, that the 
temperature is higher, but the gray values are lower. So the infrared target may be bright or dark. To judge the polarity of target is an important step in tracking specified target, if it is wrong, the subsequent segmentation is wrong too. In this paper, the method of comparing gray scale is used to judge polarity of target. To open a window $(n \times n) \mathrm{W}$, its center is the position of specified target. We judge the polarity of target through to compare the gray value of the specified target point $\left(G_{p 0}\right)$ and the gray mean of window W $\left(G_{m}\right)$, if $G_{p 0^{\prime}}>G_{m}$, the target is bright, or else is dark. If $\mid G_{p 0^{-}}$ $G_{m} \mid \leq T, T$ is a threshold, the window $\mathrm{W}$ is too small to include target, as show in Figure1 (window A), in this case, the window need to be enlarged and to do the next comparing, repeat to do until $\left|G_{p 0^{-}} G_{m}\right|>T$, as shown in Figure 1(window B)

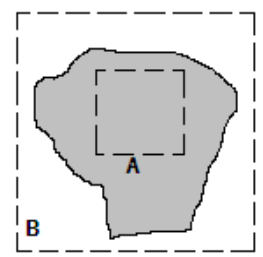

Figure 1. Window A represent that window is to small, window B is a adaptable window

\section{B. choose the seed}

In order to simplify narrative, we assume the target is bright in the follow where have no specifying. Since the target usually has a strong thermal radiation, choose the extreme point of the gray as the seed point is more reasonable, the results of region growing is stable. But for the target is specified by man, it is difficult to ensure that the specified target location is exact the extreme point. So we can not use the specified target pixel as seed directly, it need to adjust the position. According to the experience, the deviation of specified target location, should near the extreme point. Therefore, we find the brightest point in a $17 \times 17$ region, and use it as the seed. We find the brightest point in the detecting window as the seed in the subsequent frames directly.

\section{To determine the size of detection window}

After the target polarity and growing seed has been determined, target area and border information can be obtained through region growing, and then the external rectangle can be get from the target border information. We take into account the uncertainty of the moving direction and speed of target, so the actual size of detecting window should be slightly larger than the external rectangle. In the case of unknowing the size and position of detecting window, there is another problem before region growing, that is how to get the value of variable $I_{\max }$ and $I_{\min }$ in the formula (1). In this case, we assign the variables adaptive value that refer to the maximum gray value $I_{0}$. If the gray value is $\mathrm{k}$ bits, then $I_{0}=2 \mathrm{k}-1$, assign $I_{\max }=0.9 I_{0}, I_{\min }=0.1 I_{0}$.

\section{IV. . TARGET FEATURE EXTRACTION AND}

\section{REORGANIZATION}

On the complex background, often multiple targets appear simultaneously in the detecting window and be interfered each other, it bring many difficulties to segment target. Invariant features of target were widely used to overcome the difficulties. Invariant features of infrared target include area, gray value, location and shape feature, etc. There are many methods to describe shape features, such as multi-layer eigenvector (MLEV) shape descriptor[9], invariant moments[10] and Zernike moments[10, 11]. The description of the above method is in better performance, but the computation of these methods is too complex to meet the requirements of real-time in tracking.

In this paper, we proposed the spokes variance method to describe the shape feature. On the condition of meeting practical applications, the method appropriately reduce the describing performance in order to enhance the computing speed, the details as follows:

As shown in Figure 2, the gray region is the target region segmented by region growing; the point $\mathrm{C}$ in figure 2 is the centroid of the target region. To draw lines through the point $\mathrm{C}$ with the same angle $\alpha$ between it and its neighbor, the number $\mathrm{m}$ of lines can be computed by the formula $m=(180 / \alpha)+1$, the length of each line segment intercepted by the target shape as $l_{i}, \mathrm{i}=1 \ldots m$, the shape feature $F$ will be computed as follow:

$$
F=\sqrt{\frac{1}{m} \sum_{i=1}^{m}\left(l_{i}-\bar{l}\right)^{2}}, \quad \text { where } \bar{l}=\frac{1}{m} \sum_{i=1}^{m} l_{i}
$$

The small value of $F$ represent the shape is close to circle shape, the larger value represent the shape is more irregular. It is easy to understand from its theory that the algorithm has rotation invariance. The smaller value of $\alpha$ imply that the description of shape is finer, and the rotation invariance is more obvious, but the computation will increases too, its value can be determined based on the practical application. Because the point $\mathrm{C}$ is the centroid of the shape, it also has translation invariance. If the length of the line segment is normalized, it would have the scale invariance. However, in practice, the target size only have little change between the current frame and previous frame, so it have no significance to normalize the length of line segments, it will increase the amount of computation in reverse.

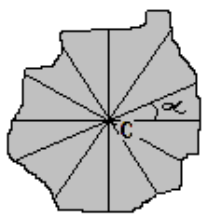

Figure 2. Example of SV method 


\section{TARGET BEING COVERED}

The target often be covered during moving on the complex background, in this case, the target is easy to lose, so it is need to predict the moving track during the target being covered, or else the target will not be capture when the target appear again. The solution is to predict the target position and orientation through the Kalman Filter. For the moving orientation possible have little change when target moving, the deviation will become larger when the time of being covered is long. In order to capture the target when it move out the obstacle, the size of detection window should be adjusted as time of target behind the obstacle, when the frame number increased, the size of the window size should be adjusted bigger too. Experimental results show that the target can be captured on the condition that the frame number of being covered less than 30 frames and the speed and orientation have little change.

\section{EXPERIMENTAL RESULT}

There are a lot of vehicles on the bridge in the figure $3 \mathrm{~A}$, we specify a target that is not the brightest one in the experiment, and there is another brighter target near it. The specified target still be tracked without being interfered with by the brighter target behind it after more than 100 frames. In the figure $3 \mathrm{~B}$,
A

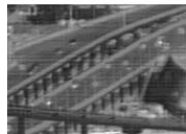

B

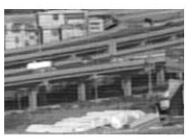

$\mathrm{C}$
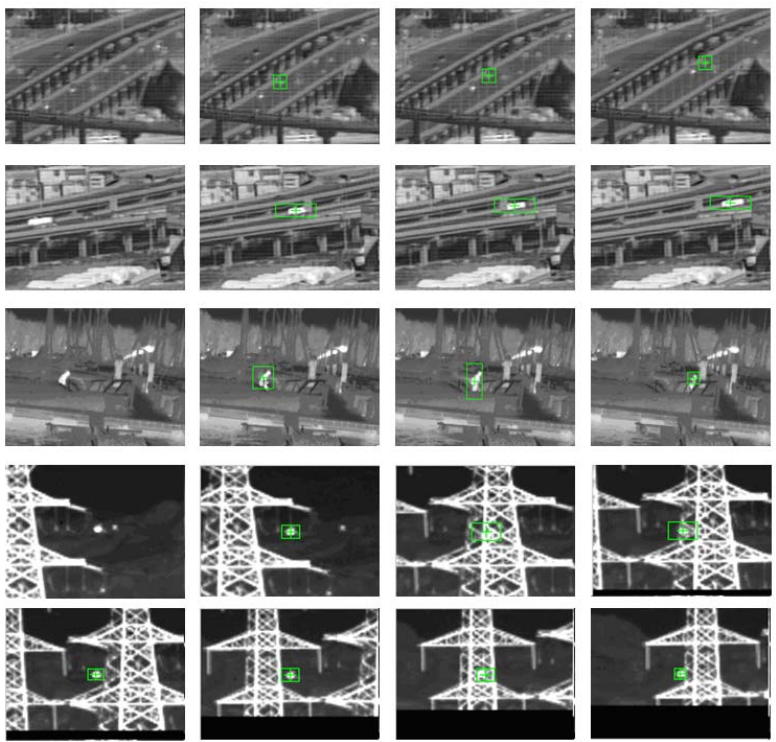

Figure 3. Experimental result, the first image in each group is an original image in video; the others are the key frames of tracking the specified target is partly covered by the traffic sign in the course of moving. The algorithm can track the target when it behind the traffic sign. In figure $3 \mathrm{C}$, we specify a person, which is a non-rigid object, as the target, its shape will change in the course of walking. The experimental result shows that the algorithm can track the target and automatically adjust the size of detection window. In figure $3 \mathrm{D}$, the power tower is more bright than the plane, it is impossible to segment the plane. In this case, the algorithm use shape feature to exclude the false target and use the Kalman Filter to predict the position of target. After the target crossed power tower, it be capture again and the window be adjusted to the target size. The experimental result show the algorithm is good.

\section{CONCLUSION}

In this paper, we analyze the problem that should be solved in tracking a specified target, and propose the reasonable method to solve them, such as to judge the target polarity by comparing the gray value, to segment the target by using region grow, to exclude the interference by using the features of target, and to solve the problem of covering target in short time by Kalman Filter. The experiments prove that the algorithm is simple and its computing speed is fast, and the tracking performance is fine.

\section{REFERENCES}

[1] Schreiber D. Robust template tracking with drift correction. Pattern Recognition Letters[J], 2007, 28(12) PP: 1483-1491.

[2] Li Zhong-jian;Du Juan;Guo Lu. Multi-threshhold segmentation and optimization based on otsu in color image. Computer Engineering and Applications[J], 2010, 46(Copyright 2011, The Institution of Engineering and Technology) PP: 176-178.

[3] Zhong Qu;Li Zhang. Research on image segmentation based on the improved otsu algorithm//Proc. of 2010 2nd International Conference on Intelligent Human-Machine Systems and Cybernetics (IHMSC 2010), 26-28 Aug. 2010[C]. Los Alamitos, CA, USA: IEEE Computer Society. 2010, PP: 228-231.

[4] Zhaoping Wu;Tao Su. Radar target detect using particle filter//Proc. of 2010 IEEE International Radar Conference, 10-14 May 2010[C]. Piscataway, NJ, USA: IEEE. 2010, PP: 955-958.

[5] Comaniciu D.;Ramesh V.;Meer P. Kernel-based object tracking. Ieee Transactions on Pattern Analysis and Machine Intelligence[J], 2003, 25(5) PP: 564-577.

[6] Shih F. Y.;Cheng S. X. Automatic seeded region growing for color image segmentation. Image and Vision Computing[J], 2005, 23(10) PP: 877-886.

[7] Park B.;Furlan A.;Patil A.;Bae K. T. Segmentation of blood clot from ct pulmonary angiographic images using a modified seeded region growing algorithm method $[\mathrm{M}]$, Medical imaging 2010: Image processing, Dawant B. M.;D. R. Haynor, EditorsBellingham: Spie-Int Soc Optical Engineering. 2010.

[8] Vieira M.;Shimada K. Surface mesh segmentation and smooth surface extraction through region growing. Computer Aided Geometric Design[J], 2005, 22(8) PP: 771-792.

[9] Kim H. K.;Kim J. D. Region-based shape descriptor invariant to rotation, scale and translation. Signal Processing-Image Communication[J], 2000, 16(1-2) PP: 87-93.

[10]Zhang D. S.;Lu G. J. A comparative study of curvature scale space and fourier descriptors for shape-based image retrieval. Journal of Visual Communication and Image Representation[J], 2003, 14(1) PP: 41-60.

[11] Wee C. Y.;Paramesran R.;Takeda F. Sorting of rice grains using zernike moments. Journal of Real-Time Image Processing[J], 2009, 4(4) PP: 353-363. 